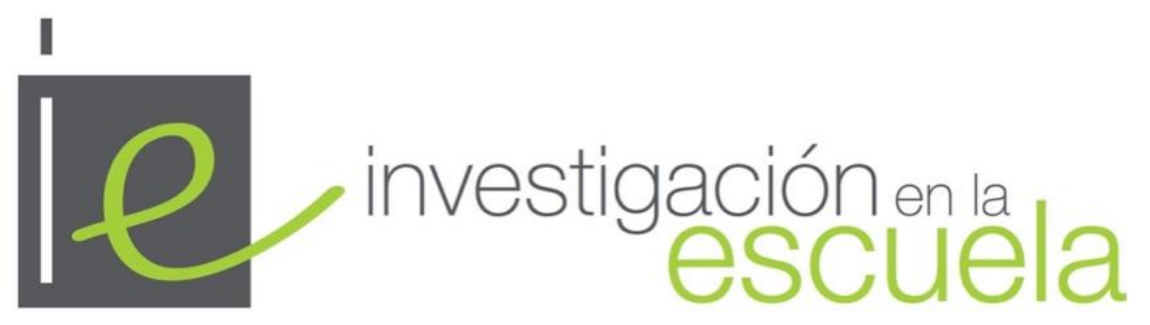

Revista de Investigación e Innovación Educativa nº 104, 2021 | e-ISSN 2443-9991

\title{
Las víctimas del conflicto armado: una comprensión desde la educación matemática
}

The victims of the armed conflict: an understanding from math education

\begin{abstract}
Mag. María Inés Cano Villamil es Magister en Docencia de la Matemática de la Facultad de Ciencia y Tecnología de la Universidad Pedagógica Nacional (Colombia)·micanov@upn.edu.co · https://orcid.org/0000-0002-77393266
\end{abstract}

Mag. Laura Ginneth López Romero es Magister en Docencia de la Matemática de la Facultad de Ciencia y Tecnología de la Universidad Pedagógica Nacional (Colombia)·llopezr@upn.edu.co·https://orcid.org/00000003-0440-7062

iD Dra. Elizabeth Torres Puentes es Profesora tiempo completo en la Facultad de Educación de la Universidad Pedagógica Nacional (Colombia)·etorresp@pedagogica.edu.co·https://orcid.org/0000-0002-3642-0571

Cómo citar este artículo

Cano Villamil, M.I., López Romero, L.G. y Torres Puentes, E. (2021). Las víctimas del conflicto armado: una comprensión desde la educación matemática. Investigación en la Escuela, 104, 65-79. doi: http://dx.doi.org/10.12795/IE.2021.1104.06

Resumen. Este artículo es producto de una investigación en el marco de la Maestría en Docencia de la Matemática en la Universidad Pedagógica Nacional (Colombia). El objetivo de la investigación fue aportar desde el diseño, gestión y evaluación de un Escenario de Investigación, generado en el marco de la Educación Matemática Crítica, a la comprensión de algunas modalidades de violencia del conflicto armado colombiano que ha impactado a las víctimas, y a la formación en competencias matemáticas en un grupo de estudiantes de educación media. Para efectos de este artículo se retoman los resultados correspondientes a la fase de gestión del Escenario de Investigación, Escenario de Investigación diseñado por maestrantes e implementado con estudiantes de grado décimo, el cual se basó en el estudio de una modalidad de violencia presente en el conflicto armado colombiano por medio del análisis y reflexión de diferentes datos e información verídica sobre las víctimas. Una de las conclusiones del estudio que derivó este artículo enfatiza en la necesidad de hacer de la educación matemática, una herramienta potente, que aporte a la comprensión de las realidades sociales, tal como lo es el conflicto armado colombiano. Otra conclusión refiere a la importancia de la gestión de Escenarios que permitan promover las competencias matemáticas para ciudadanos, que lleven a una conciencia profunda de lo que significa el lugar de las víctimas, en el marco de la guerra.

Abstract. This article is the product of an investigation within the framework of the Master's Degree in Mathematics Teaching at the National Pedagogical University (Colombia). The objective of the research was to contribute from the design, management and evaluation of a Research Scenario, generated within the framework of Critical Mathematics Education, to the understanding of some modalities of violence of the Colombian armed conflict that has impacted the victims, and training in mathematical competencies in a group of high school students. For the purposes of this article, the results corresponding to the management phase of the Research Scenario are retaken, Research Scenario designed by teachers and implemented with tenth grade students, which was based on the study of a modality of violence present in the Colombian armed conflict through the analysis and reflection of different data and truthful information about the victims. One of the conclusions of the study that derived this article emphasizes the need to make mathematics education a powerful tool that contributes to the understanding of social realities, such as the Colombian armed conflict. Another conclusion refers to the importance of the management of Scenarios that allow promoting mathematical competencies for citizens, leading to a deep awareness of what the place of the victims means, in the context of war. 
Palabras clave $\cdot$ Keywords

Conflicto, violencia, ciudadanía, educación para la paz, educación matemática, educación crítica. Conflict, violence, citizenship, peace education, mathematical education, critical education.

\section{Introducción y estado de la cuestión}

Para dar cuenta del estado de la cuestión se ubicaron algunas investigaciones recientes que trabajaron la relación entre pensamiento crítico y/o político y las matemáticas, aunque vale decir que no se encontraron trabajos que desarrollaran específicamente la relación entre educación matemática y conflicto armado.

Un primer grupo de trabajos refieren a la estadística crítica. Allí están las investigaciones de Ayala (2019), Álvarez y Montoya (2011), González y Tovar (2017) y Zamora (2018) cuyo punto en común es el enfoque de Educación Estadística Crítica definido por Campos (2007, citado por Álvarez y Montoya, 2011) como la convergencia entre la Educación Estadística, la Educación Crítica y la Educación Matemática Crítica, con el fin de "proveer de significado a los contenidos estadísticos de forma democrática, motivando en los estudiantes el desarrollo del espíritu crítico, la responsabilidad ética y la concientización política" (p. 21). Particularmente las propuestas de Ayala (2019) y González y Tovar (2017) muestran un primer acercamiento hacia la formación ciudadana y la educación estadística para la interpretación y comprensión de aspectos propios del conflicto armado, foco de interés de las autoras de este artículo.

Un segundo grupo refiere a las fortalezas y debilidades en la implementación de un ambiente de aprendizaje, donde se ubican las investigaciones de Amaya (2018), Chaparro (2019), Angulo y Solano (2013), y Torres (2016). Estos trabajos reflexionan sobre aspectos que están presentes durante la implementación de un Ambiente de Aprendizaje diseñado desde la perspectiva de la Matemática Crítica.

En un tercer grupo se puede situar la investigación de Gómez (2016), el cual propende por la construcción de ciudadanías ambientales desde las prácticas de medición y la construcción de un espacio de reforestación, en relación con las matemáticas.

De acuerdo con el estado de la cuestión, la investigación de la que se deriva este artículo partió de dos tensiones. La primera se expresa en la escasa visibilidad del conflicto armado en la escuela como asunto de reflexión curricular, lo que implica que poco se dialogue sobre al asunto, impidiendo la formación crítica y política de los estudiantes.

Al respecto, Pagés (2009) y Arias (2015) consideran que el problema principal no es discutir sobre la ampliación de las temáticas en el currículo de ciencias sociales, sino en las finalidades de este conocimiento y su análisis para la formación ciudadana, en este caso, el reconocimiento de las víctimas del conflicto armado, identificando los derechos humanos y constitucionales que le son vulnerados y las consecuencias que esto trae para la sociedad colombiana. Pese a que en la escuela no se propicia la reflexión en profundidad de las modalidades de violencia y lo que esto ha implicado para las víctimas, Torres (2015), Lizarralde (2012) y Romero (2012), manifiestan que la relación entre el conflicto armado y escuela se ha dado de manera cruenta, pues esta se ha usado como trinchera, campamento, helipuerto, parqueadero de tanques de guerra, escenario para matar y espacio físico para crímenes de lesa humanidad; según estos autores esta institución no ha sido un lugar para la enseñanza o la protección de la infancia.

Como segunda tensión se establece la no evidente relación, al menos para la escuela, entre enseñanza de la matemática y conflicto armado, particularmente la comprensión de algunas modalidades de violencia, de forma que se suscite la formación de ciudadanos críticos. Esa falta puede darse, de acuerdo con Valero (2006) y Millán y Vernaez (2005), porque la enseñanza de las matemáticas se da de una manera poco contextualizada, alejada de las realidades de los estudiantes, que, para el caso de Colombia, puede ser el drama del conflicto armado.

En ese sentido, la educación matemática debe propender por el alcance de la democracia en sociedades tan desiguales e injustas como lo es la colombiana. Así, Skovsmose y Valero (2012), reconocen que la relación entre matemáticas y democracia es posible dado que: hay una resonancia intrínseca que dota a las matemáticas de poder y permite a las personas adquirir grandes potencialidades para su vida, siempre desde aspectos positivos que destacan los avances tecnológicos y científicos; hay disonancia, en tanto las matemáticas se constituyen como un mecanismo de exclusión y estratificación dentro del aula; hay una relación crítica, mediante la cual se establece que, dependiendo de las prácticas y el sentido que se le da a las matemáticas y a su enseñanza se puede afectar o no la consolidación de la democracia y la justicia social.

A partir de lo anterior se puede afirmar que a las matemáticas se les debe dar el carácter de herramienta que ayuda a la comprensión de situaciones políticas, sociales y económicas, puesto que ellas favorecen la argumentación, comunicación, razonamiento, resolución de problemas, entre otros procesos fundamentales que permiten entender distintos fenómenos actuales. 


\section{Material y método}

Para efectos de este artículo, se presentan los resultados relacionados con las producciones de un grupo de estudiantes de educación media, en el marco de la gestión de un Escenario de Investigación, con el cual se pretendió desarrollar competencias matemáticas del ciudadano (Alsina, 2010), a propósito de la comprensión de algunas modalidades de violencia del conflicto armado colombiano. En atención a ello se exponen las categorías de orden teórico y metodológico para abordar ese objetivo.

\subsection{Consideraciones teóricas}

Desde la formación ciudadana crítica se puede aportar a la compresión del conflicto armado colombiano. Dicha formación se vincula al desarrollo de competencias ciudadanas, entendidas como "el conjunto de capacidades y habilidades cognitivas, emocionales y comunicativas -integradas- relacionadas con conocimientos básicos (contenidos, procedimientos, mecanismos) que orientan moral y políticamente nuestra acción ciudadana" (Ruíz y Chaux, 2005, p. 32), y se adopta la propuesta del Ministerio de Educación Nacional -MEN- (2006), en la cual se reconoce la importancia de todas las áreas del conocimiento en la formación ciudadana, de forma que se suma el papel de la educación matemática y los aportes que desde esta se pueden hacer para este propósito.

Millán y Vernaez (2005) plantean que la enseñanza tradicional de las matemáticas se aleja del desarrollo de prácticas críticas relacionadas con principios democráticos, en tanto la primera da valor a procesos algorítmicos e irreflexivos y se centra en realidades artificiales que impiden ver las matemáticas como herramienta para evidenciar desigualdades e injusticias. La matemática crítica como herramienta se convierte en práctica humana de valor social y político que permite impartir juicios y argumentos de verdad, que influyen en el desarrollo personal de un estudiante, permitiéndole reconocer la importancia de su participación democrática e interacción con la sociedad, desde acciones fundamentadas en sus conocimientos, para definir, transformar y cambiar sus realidades y las del mundo (Gutiérrez, 2013).

En este sentido, Alsina (2010) manifiesta que los estudiantes pueden llegar a ser ciudadanos libres y reflexivos siendo matemáticamente competentes al aplicar la disciplina a su vida diaria, es decir, ciudadanos capaces de realizar tareas elementales como cobrar, pagar, entender recibos, analizar gráficas en periódicos, interpretar porcentajes; tareas saludables como entender la química de los alimentos, leer resultados clínicos de sangre, los intervalos de referencia en diferentes imágenes médicas, las dosis de medicamentos, diagnósticos; tareas consumistas como el buen uso de la tarjeta de crédito, las ofertas, rebajas, impuestos o alternativas de pago que requieran toma de decisiones con el mejor criterio; y tareas democráticas como entender encuestas, muestras, sondeos, leyes electorales, temas relacionados con financiación y fiscalidad. De forma general, el autor expresa que las matemáticas preparan al estudiante para realizar algunas acciones básicas convivenciales como entender alternativas, aclarar conceptos, tomar decisiones, comparar modelos, planificar a largo plazo, interpretar información, dialogar inteligentemente, resolver problemas, defender principios, desarrollar la creatividad, razonar y argumentar.

Con lo anterior, se entiende la competencia matemática como "la habilidad de entender, juzgar, hacer y usar matemáticas en una gran variedad de situaciones y contextos en los cuales la matemática juega o podría jugar un papel importante" (Niss citado en Alsina, 2010, p. 94). Alsina (2010) describe las competencias matemáticas para el ciudadano así:

1. Pensar matemáticamente: Aplicar los conocimientos matemáticos a la realidad y cotidianidad, por medio del pensamiento cuantitativo y lógico, con el fin cuestionar la información. Esto implica entender conceptos, abstraer, intuir, relacionar, generalizar y criticar.

2. Razonar y argumentar matemáticamente: Aportar razones o argumentos propios mediante deducciones o demostraciones genuinas, que se validan desde el rigor disciplinar y/o el sentido crítico de los razonamientos cotidianos.

3. Resolver problemas: Determinada como una actividad implícita o explícita en la vida social, que permite poner a prueba la relación que se establece entre el conocimiento matemático escolar y la cotidianidad.

4. Saber hacer modelos: Capacidad de matematizar el mundo, al poder definir modelos desde la realidad, y así mismo poder analizar el modelo para aplicarlo.

5. Comunicación: Poder expresar y entender ideas por medio de diferentes recursos expresivos, como gráficos cuantitativos o cualitativos.

6. Representación: Representar o expresar objetos y situaciones mediante palabras, símbolos, números, historias, material o dibujos. 
7. Usar símbolos: Uso correcto de simbología, tanto en lenguaje natural como en formalismos, para que no se presenten confusiones.

8. Uso adecuado de instrumental: Consiste en utilizar adecuadamente instrumentos manuales y tecnológicos.

En esta dirección, el uso de las competencias matemáticas para ciudadanos en la comprensión de algunas modalidades de violencia presentes en el conflicto armado colombiano permite reconocer la realidad que nos ha dejado la guerra, como consecuencia del pasado. Así mismo, abre caminos para generar acciones o estrategias de cambio a las realidades de futuros lejanos o cercanos, siempre con miras a una sociedad en paz. Esto teniendo en cuenta que todas las acciones realizadas por el individuo generan conocimiento y ofrecen la capacidad de explicar, manejar y entender la realidad, aportando memorias individuales y colectivas a las distintas generaciones, con el fin de no repetir el ciclo vital en el que la realidad depende inexorablemente del pasado (D'Ambrosio, 2007).

El desarrollo de competencias matemáticas contribuye a la consolidación de sujetos críticos, los cuales se caracterizan por cuestionarse sobre lo que sucede a su alrededor, problematizar algunas situaciones de impacto en la comunidad y entender cómo actuar sobre estas. Para Skovsmose (1999) el ser crítico "significa prestarle atención a una situación crítica, identificarla, tratar de captarla, comprenderla y reaccionar frente a ella" (p. 16), entendiendo situación crítica como fenómenos de: represión, conflicto, contradicción, miseria, desigualdad, devastación ecológica y explotación. Esta actitud permite a los estudiantes alcanzar una visión transformadora de su entorno, que contribuye al avance y la superación de problemas políticos, sociales, ambientales o económicos.

De forma particular, desde la educación matemática, se reconoce la perspectiva de la Educación Matemática Crítica, caracterizada por el interés sobre los roles socio-políticos, positivos o negativos, que desempeñan las matemáticas en la realidad y cómo la educación matemática puede contribuir con ello (Skovsmose citado en Scaglia, 2012). Una forma de relacionar la educación matemática con aspectos sociopolíticos se puede ver a partir de la equidad, la cual en ámbitos educativos busca que todos los estudiantes reciban el mismo trato, gocen de un currículo flexible y sensible a la diversidad cultural presente en el aula, y logren el alcance de metas comunes (UNESCO citado en Scaglia, 2012).

Skovsmose y Valero (citados en Scaglia, 2012) describen la relación entre equidad y educación matemática como crítica, pues solo los conocimientos matemáticos no son suficientes para que las personas se "empoderen" y apoyen la justicia social, dado que las matemáticas también los pueden "desempoderar" para avalar posiciones autoritarias o de exclusión. Además, consideran que la educación se ha encargado de formar individuos meramente "consumidores", que pueden realizar diferentes acciones económicas, ambientales y hasta aparentemente democráticas, al ejecutar mecánicamente normas de un orden social ya establecido, pero no son capaces reflexionar sobre su accionar ni de ejercer la ciudadanía más allá de lo estipulado.

\subsection{Consideraciones metodológicas}

En la investigación que da lugar a este artículo, se consideró apropiado el enfoque cualitativo, pues se trabajó con población escolar y se ponderó la interacción escolar en un espacio social determinado, por otro lado este enfoque fue el más acorde con la temática seleccionada, ya que el conflicto armado colombiano es una problemática que ha dejado afectaciones sociales, atentando contra los derechos humanos a través de las modalidades de violencia establecidas por el Grupo de Memoria Histórica -GMH- (2013). Además, se enmarcó en un diseño de Investigación Acción que, según Stringer (citado en Hernández, 2014), se caracteriza por combatir la injusticia social, ser democrático y equitativo, en el que todos los miembros del grupo pueden participar y sus aportes son valorados y determinantes para que la comunidad busque mejorar sus condiciones de vida.

De acuerdo con lo anterior se propuso desarrollar un Escenario de Investigación, cuya referencia es una situación de la vida real (Skovsmose, 2000) configurado en tres fases: diseño, gestión y evaluación. Este artículo se centra en la segunda. En dicha fase las categorías de análisis se construyeron a la luz de las competencias matemáticas para el ciudadano propuestas por Alsina (2010), tal y como se recoge en la tabla 1. 
Tabla 1

Categorías de análisis Competencias matemáticas para el ciudadano. (Construcción propia a partir de Alsina, 2010)

Competencias Matemáticas en relación con la comprensión del conflicto armado colombiano

A. Pensar matemáticamente: Aplicar los conocimientos matemáticos para comprender algunas modalidades de violencia del Conflicto Armado colombiano o cuestionar la información proveniente de noticias, informes, testimonios, documentales o películas que se relacionan con las mismas.

B. Resolver problemas: Actividad implícita o explícita que permite poner a prueba la relación que se establece entre el conocimiento matemático escolar y la compresión del conflicto armado colombiano.

C. Comunicación: Entender o presentar ideas por medio de recursos expresivos como gráficos cuantitativos o cualitativos.

D. Uso adecuado de instrumental: Utilizar adecuadamente instrumentos manuales y tecnológicos para organizar y representar la información.

E. Usar símbolos: Uso correcto de simbología que manifieste sus ideas e interpretaciones y no se presenten confusiones.

F. Razonar: Aportar razones o argumentos propios que se sustentan en la información consultada y el análisis de esta, teniendo en cuenta el rigor disciplinar y el sentido crítico de los razonamientos cotidianos.

G. Representación: Representar o expresar situaciones o reflexiones de las afectaciones generadas por las modalidades de violencia a través de recursos verbales o visuales como palabras, símbolos, números, historias, material o dibujos.

La propuesta fue diseñada para ser desarrollada con estudiantes de grado décimo de un colegio privado de Bogotá, cuyas edades oscilan entre los 16 y 18 años. Se diligenciaron los respectivos consentimientos informados con los padres de familia. El grupo de estudiantes no es homogéneo en cuanto a que los intereses de algunos no se centran en las matemáticas.

\section{Análisis y resultados}

Las evidencias aquí presentadas hacen parte del producto final entregado por los estudiantes en la fase de gestión, el cual consistía en una página web donde, por grupos, plasmaron sus análisis y reflexiones, dicha página se puede consultar en el enlace https://lopezlao1.wixsite.com/edumat-catpaz. Adicionalmente, se extraen evidencias de las transcripciones de audio y vídeo obtenidas durante las sesiones de clase desarrolladas en la fase de gestión.

En lo relacionado con la competencia Pensar matemáticamente, se evidenció con frecuencia que la cifra de víctimas como cantidad numérica generaba impacto en los estudiantes y hacía un llamado para la toma de conciencia frente a la gravedad de la problemática, tal como se percibe en la opinión de la estudiante 16 (Figura 1).

\section{Figura 1}

Categoría pensar matemáticamente - Transcripción de audio 8 de junio

Grupo 5- transcripción audio 8 de junio de 2020

Estudiante 16: [...] eh también lo que decía [17], como todas esas cifras tan grandes que daban de desplazados, de desaparecidos, de secuestros, a uno también lo hace como caer en cuenta de la realidad porque uno dice como a bueno "x un desaparecido", por ejemplo, en noticias o cosas asi, pero digamos cuando uno ya lee la cantidad total uno dice iush esto si es grave!, o sea, enserio que este país esta como, como lo decían en el documental desangrándose.

El énfasis que realiza la estudiante sobre las cifras es un proceso en el cual identifica una desigualdad numérica entre la cantidad total de víctimas de las modalidades de violencia y la cantidad de víctimas que ella consideraba existían. El cuantificar correctamente la cantidad de víctimas le aportó en la comprensión de la complejidad del conflicto armado.

Por otro lado, cuando la estudiante hace alusión a la cantidad de desaparecidos se reconoce que los estudiantes conocen casos aislados de las víctimas por medio de noticias, lo que confirma que pese a que este tipo de datos aparecen en los medios de comunicación, suelen ser presentados de forma desligada y descontextualizada, lo que dificulta a los espectadores establecer relación con una única problemática que les permita dimensionar correctamente la cantidad de víctimas, siendo esta la causa del impacto de la estudiante al momento de conocer las cifras reales. Al respecto el GMH (2013) afirma que "el contexto de 
violencia generalizada en el que discurre el conflicto es aprovechado por los actores armados legales e ilegales para invisibilizar sus acciones y confundirlas con hechos violentos perpetrados por otros" (p. 33).

En lo que respecta a la competencia Resolver problemas, se evidencia como la lectura de gráficos de variables cualitativas o cuantitativas describen claramente aspectos específicos de cada modalidad de violencia. El grupo 7 organizó los datos sobre reclutamiento ilícito según los años, construyendo un gráfico de líneas que no representaba correctamente los datos, la estudiante 23 al realizar la lectura de la gráfica se percató del problema.

En el diálogo la estudiante 22 se percata que el gráfico no tiene títulos, sin embargo, no tiene en cuenta que los datos graficados no representan correctamente la información, es decir, no se hizo una lectura correcta de la información representada en el mismo. Es la estudiante 23 quien se fija en la forma de la gráfica, reaccionando y manifestando a sus compañeras que lo que está ahí no tiene coherencia porque, como ella lo expresa, "no todos los años pueden subir", puesto que una línea recta le indicaba que existía un incremento a lo largo de los años de forma constante, y según lo proporcionado en la base de datos estas cantidades varían año a año, como se puede evidenciar en la gráfica que construyeron después de corregir este error (Figura 2).

\section{Figura 2}

Categoría resolver problemas - Transcripción de audio 13 de julio y página web

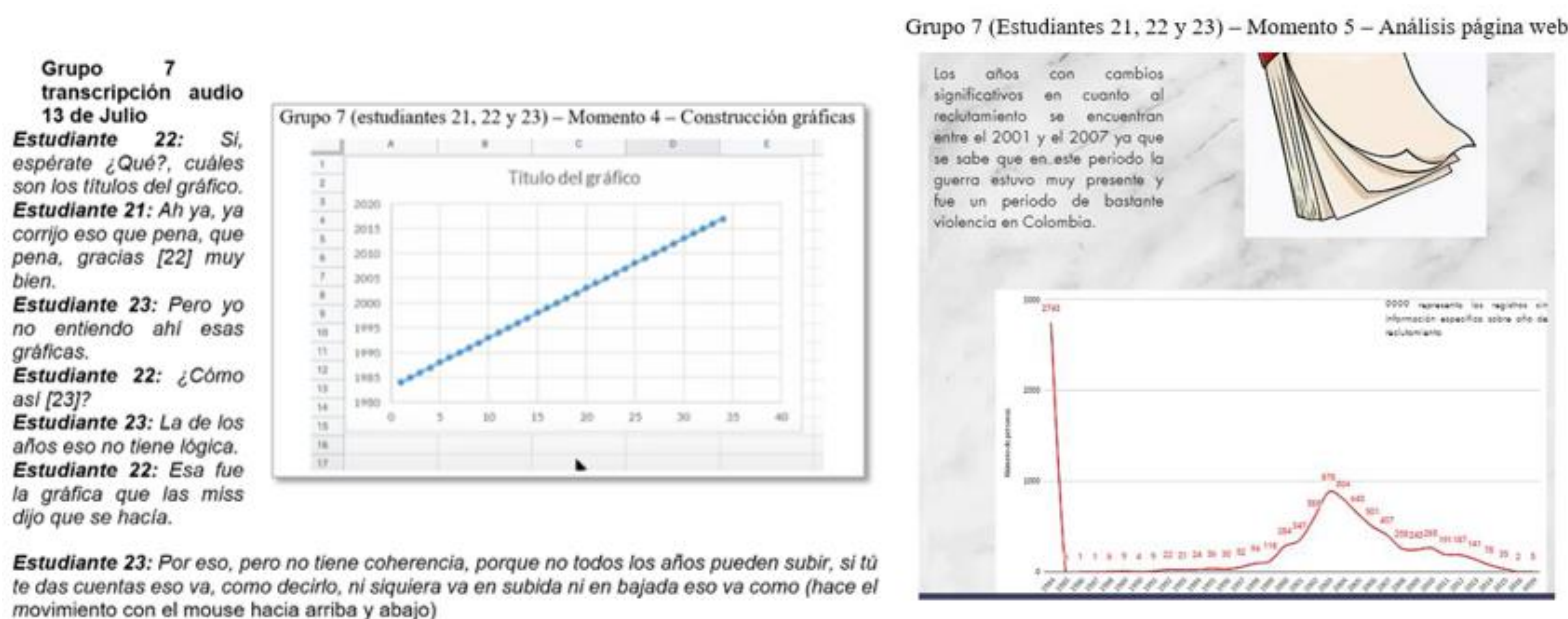

Esta situación resalta la importancia de la lectura adecuada de gráficas lineales, ya que al dar sentido a su comportamiento en relación con el incremento o disminución del número de víctimas, tal como lo realizó la estudiante 23, fue posible corroborar que existía un error en la información que estaban representado, es así como para su análisis final lograron generar la gráfica correcta (Figura 2) y realizar la lectura al gráfico en el que es posible asociar un periodo de años con notables incrementos y disminuciones con una época de violencia muy marcada en el país, puesto que según el documental "No hubo tiempo para la tristeza", que habían visto en sesiones anteriores, se exponía que aproximadamente entre 1995 y 2005 hubo un recrudecimiento del conflicto armado colombiano.

La relación que ellas establecen entre la información del documental y la información de la gráfica es evidencia de la vinculación entre la información que reciben de diferentes medios. Skovsmose y Valero (2012) afirman que estos vínculos emergen porque "las competencias en una disciplina interactúan con las competencias desarrolladas en otras disciplinas" (p. 17), en este caso las competencias matemáticas están centradas en el uso de sus conocimientos para comprender el reclutamiento ilícito, específicamente las estudiantes utilizaron sus saberes sobre el conflicto armado para justificar porqué se da el comportamiento en el gráfico de líneas, lo cual les permite realizar comprensiones más profundas con razonamientos como a mayor violencia entre los grupos armados mayor número de víctimas del reclutamiento ilícito y viceversa.

En relación con la competencia matemática del ciudadano Comunicación, se resalta que los estudiantes de los 7 grupos de investigación basaron sus presentaciones finales en cifras e información consultada, lo cual les permitió comunicar aspectos específicos de las víctimas de cada modalidad de violencia como edad, años, departamentos, estado físico, entre otros.

Comunicar ideas y clarificaciones a través de gráficos estadísticos fue la herramienta que más utilizaron los estudiantes en exposiciones respecto a cada modalidad de violencia, por ejemplo, las estudiantes del 
grupo 7 a partir de un gráfico de barras representaron la pertenencia racial de víctimas del reclutamiento ilícito (Figura 3).

\section{Figura 3}

Categoría comunicación - Página web

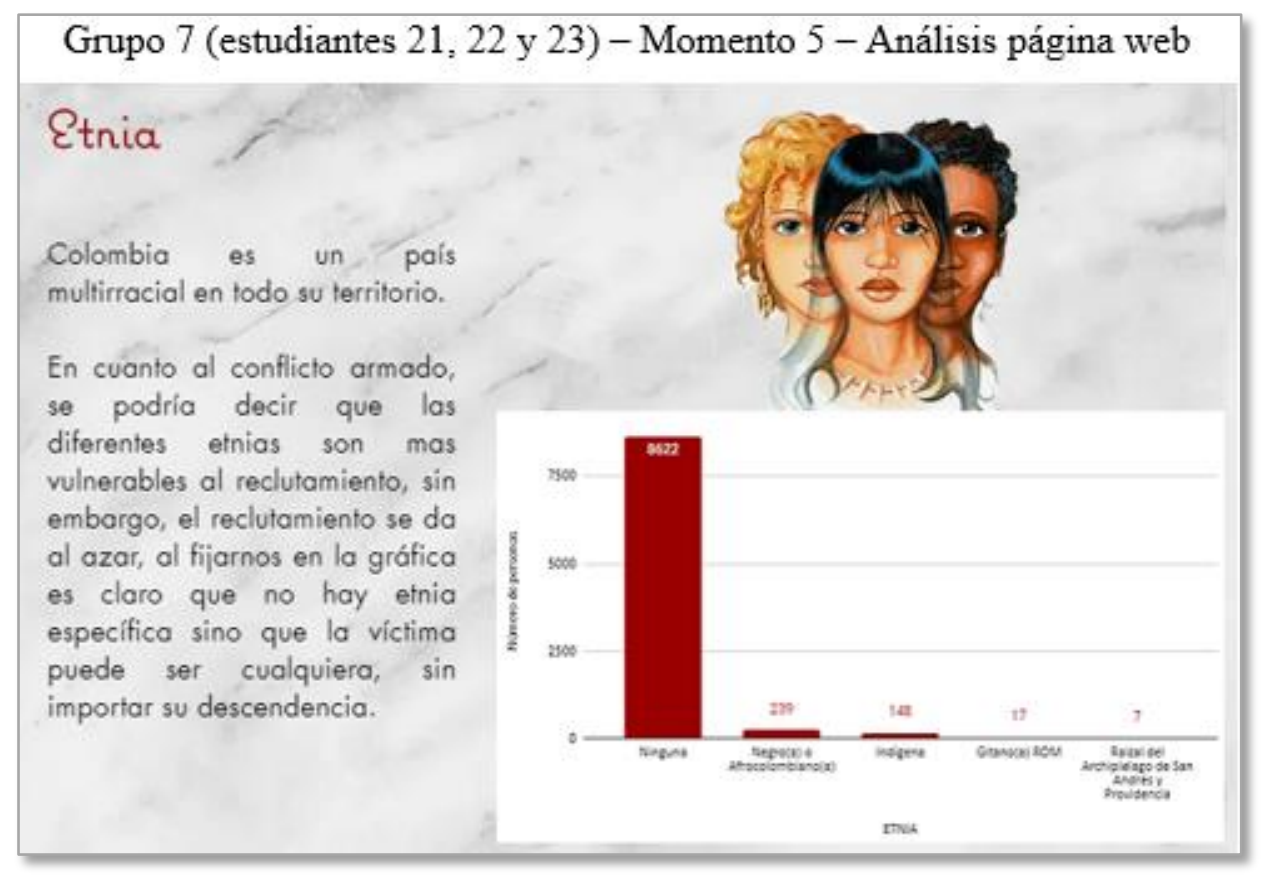

Inicialmente, el grupo 7, realiza una introducción en la que comunica cómo la percepción respecto a la característica de Colombia como país multiétnico había permeado su idea sobre el tipo de personas que son reclutadas, asumiendo que determinados grupos podían ser más vulnerables a esta modalidad de violencia, sin embargo, manifiestan que "al fijarnos en la gráfica es claro que no hay etnia específica", es decir, las estudiantes se dan cuenta que esta característica no es relevante. En este caso la gráfica de barras fue una herramienta que les permitió comunicar porqué cambiaron su percepción frente a la vulnerabilidad que consideraban tenía algunas comunidades, por lo tanto, concluyen que el riesgo de sufrir esta modalidad depende de otros factores diferentes a la etnia, o como ellas lo denominan "es al azar", "la víctima puede ser cualquiera".

Respecto a la competencia referida al Uso adecuado de instrumental, se destaca que los estudiantes tuvieron la oportunidad de acceder a diferentes recursos tecnológicos como hojas de cálculo, presentación con diapositivas y documentos de Google que ofrecen la posibilidad de modificarlos en línea por distintas personas al mismo tiempo, lo cual facilitó el trabajo en equipo, además del uso de la plataforma Wix para la creación de su página web; el uso de estas herramientas les permitió organizar y representar la información obtenida en la película Pequeñas voces, las noticias buscadas por internet, el documental No hubo tiempo para la tristeza, apartados del informe Basta Ya, y las bases de datos del Registro Nacional de Víctimas RUV- o la Dirección para la Acción Integral Contra las Minas Antipersonal -Daicma-.

En el proceso de organización y sintetización de los datos extraídos del RUV y DAICMA se evidenció que los estudiantes no tenían total manejo de las herramientas tecnológicas, como las hojas de cálculo, en las cuales los estudiantes discriminaron los datos de cada modalidad de violencia, generaron tablas y realizaron las gráficas que presentaron en su página web. Por ejemplo, el caso del grupo 5, quienes para discriminar los años registrados por las víctimas realizaban procesos manuales como revisar toda la base de datos e identificaban los años que debían tener presente en el conteo de víctimas (Figura 4 - Grupo 5). 


\section{Figura 4}

\section{Categoría uso adecuado de instrumental - Transcripción de audio y vídeo 6 de julio}

Grupo 5 - Momento 4 - transcripción audio 6 de julio

Estudiante 16: No entiendo cómo voy a hacer eso del año, hay como 102 años.

Estudiante 17: pero qué es lo que toca mirar ¿llos años?

Estudiante 16: Sí, toca organizarlos, pero uy no, uy nooo; o sea ahi si no sé.

Estudiante 17: Es que hay muchos años, hay 2014, 1990.

Estudiante 16: Es que por ejemplo $\mathrm{mmm}$ será que lo ponemos en rangos por ejemplo 1990 hasta 2000.

Estudiante 16: Uy es que si hay muchos.

Estudiante 17: Y de 2000 hasta 2013 y ya (risas de fondo).

Estudiante 16: Pues 1990 es como el más pequeño y el más grande

es... después de 1990 que año sigue, o sea que estén en esa cosa.

Estudiante 18: 1991 ¿luego?

Estudiante 16: 1992, 1993, 1994, 1995.

Estudiante 17: Mor pero ¿eso si está?

Estudiante 16: ¿Qué?

Estudiante 18: O sea todos esos años.

Estudiante 17: ah sí qué horror.

Estudiante 18: Bueno 95

Estudiante 16: 96, 97, 98, 99 hasta el 2000 o sea todo va como

seguido, 2001, 2002. (Se movieron sobre toda la base de datos intentado visualizar todos los años).

Estudiante 18: ¿2000 también?

Estudiante 16: Dos cero cero uno, dos cero cero dos, acabo de aprender como escribir los años wuau.

Estudiante 16: 2005, sabes esto va re largo wey. 6 también, 7, 8, 9, yo

creo que hasta el 13.

Estudiante 18: 14 también hay. ¿cómo se escribe 2010?

Estudiante 16: dos cero uno cero.

Estudiante 18: ah ya.
Estudiante 16: 16, ¿15? 16, 17, 18, hasta el 2019

Estudiante 18: Espérate que escribi mal el 2011 escribi 2110.

Estudiante 18: ¿hago todos los años del 90 al 19?

Estudiante 19: listo dale.

Estudiante 18: 2013 ¿cómo se escribe?2014, 2016, 2017.

Estudiante 16: Ah! jtambién hay 20!

\section{Grupo 3 - Momento 4- transcripción vídeo 6 de julio}

(Cuando el estudiante 10 realizó el conteo de víctimas en San Andrés y Providencia el valor que obtuvo fue cero, sin embargo, no se convenció y rectificó la escritura del comando, pero seguía siendo cero, adicional revisó toda la lista y se dio cuenta que existian datos denominados "sin información", así que decide adicionar esa casilla).

\section{Grupo 3 - Momento 4- transcripción audio 13 de julio}

Estudiante 10: Miss ya lo hice y el único comando que no me paso fue San Andrés y Providencia porque no vi nada que dijera San Andrés y Providencia, o sea, no vi ninguna, o sea, el comando lo boto como si fueran cero casos y también coloque los que eran "sin información" y todos los departamentos, voy a empezar a hacer el de género.

Estudiante 10: (realiza el total de género).

Docente: Compara a ver si es el mismo que te dio al otro lado (haciendo referencia al total de víctimas según departamentos y género).

Estudiante 10: No, no es el mismo. Creo que es San Andrés miss porque no sé cómo aparece San Andrés.

Docente: Ve a los datos, dale ahí (refiriéndose a la pestaña de filtro que acompaña el título de departamento)

Estudiante 10: Ah mira es Archipiélago.

Docente: Tienes que contarlo así Archipiélago de San Andrés y hay también Bogotá, Bogotá lo separa como algo distinto. Estudiante 10: Lo cambio y ahora si me van a dar los datos correctos. Vuelvo y lo sumo, espera ya me sale el de San Andrés, Si ya me sale.

Docente: Agrégale Bogotá porque también pone Bogotá.

Estudiante 10: Miss yo los puse absolutamente (haciendo referencia a los departamentos), ah Bogotá, obviamente.

Las estudiantes del grupo 5 reconocen que la base está compuesta por un conjunto extenso de datos y por ello pensar en agruparlos en rangos es una solución matemática práctica que habría permitido identificar fuertes periodos de violencia del conflicto armado, sin embargo, deciden pasar por alto esta idea. Adicionalmente, se logra ver que el proceso manual de búsqueda realizado por las estudiantes se da por no conocer las herramientas brindadas en la hoja de cálculo, en este caso el filtro, lo cual puede ser consecuencia de la poca experiencia que tienen con estas herramientas. En relación con lo anterior, Goñi (2010) afirma que los métodos tecnológicos premodernos de "papel y lápiz" entraron en crisis ante el uso de tecnologías electrónicas que influyen constantemente en la sociedad, por tanto, se resalta el papel de la escuela como uno de los principales lugares que debería brindar esta oportunidad, ya que estas hojas de cálculo no solo son más eficientes en cuanto agilizan la búsqueda de los datos, sino que también son más eficaces porque discriminan correctamente la información, permiten hacer conteos exactos y facilitan la construcción de los gráficos estadísticos.

El grupo 3 también discriminó los datos sin ayuda de herramientas tecnológicas, por ello durante la organización de datos respecto a los departamentos percibieron que hacía falta los registros referentes a San Andrés y Providencia, ya que en su búsqueda manual no lograron encontrar datos relacionados con este departamento (Figura 4 - Grupo 3).

En el proceso de discriminación y tabulación una de las docentes interactúa con el grupo 3 (Figura 4 Grupo 3), ayudándolos a determinar la información faltante a través de la herramienta filtro y de esta forma verificar si las tablas que habían construido eran correctas. Aunque las docentes se percataron de dar a los estudiantes los comandos necesarios para que ellos lograran contar los registro de víctimas según los aspectos ofrecidos en la base de datos, explicando en qué consistían y cómo era su escritura correcta, no se tuvo en cuenta el uso de herramientas más simples como filtrar, añadir filas o columnas, modificar las características de las gráfica o incluso la vinculación de las hojas de cálculo con la presentación de diapositivas, sin embargo, durante el desarrollo del Escenario se evidencia que los estudiantes interiorizan fácilmente el uso de herramientas tecnológicas solo si se necesitan en actividades que les exijan el uso de ellas, no por indicación de los profesores sino por necesidad, como en este caso el manejo de bases de datos extensas con información verídica sobre víctimas de las modalidades de violencia. Así como lo afirma Goñi (2010), debemos generar una revolución digital que desarrolle otro tipo de habilidades de cálculo y aproveche sin límites las herramientas tecnológicas con las que contamos en este momento.

Respecto a la competencia para el ciudadano que refiere al Uso de símbolos, se evidencia que en la página web de cada grupo se diseñaron presentaciones finales sobre las modalidades de violencia, las cuales, 
por medio de objetos matemáticos como gráficas, porcentajes, tablas de frecuencia y cifras, manifiestan las interpretaciones de los mismos a partir de sus conocimientos sobre cada modalidad.

Dado que las hojas de cálculo construyen gráficas a partir de la tabulación, los estudiantes son quienes deben determinar cuál es el gráfico que manifiesta, expresa o presenta correctamente la información y sus conocimientos frente a la modalidad de violencia. El grupo 4 se dio cuenta que, en el gráfico de barras sobre el reclutamiento ilícito según su género parecía que no existieran registros relacionados con víctimas LGBTI aun cuando había 2 víctimas (Figura 5 - Grupo 4).

\section{Figura 5}

Categoría usar símbolos - Transcripción de audio 21 de julio y página web

Grupo 4 - Momento 4 - transcripción audio de 21 de julio Estudiante 13: Espérame Miss no me marca uno.

Docente: ¿Cuál LGBTI?

Estudiante 13: Si.

Docente: Es porque son muy poquitos ¿solo son dos?

Estudiante 13: Aja.

Docente: Espérate a ver

Estudiante 13: Yo pensé que si lo hacia más grande se vería ahi pero no. (agranda la gráfica)

Docente: [...] Dale clic afuera a ver si me carga, ya es que cree una copia.

Estudiante 13: „Ya mira! no, pero es que mira hay un poco de datos que ni siquiera me muestra. (Aumenta y disminuye el tamaño de la gráfica).

Docente: Es que son muy poquitos entonces no alcanzan a mostrarios, pero hay una cosa donde dice personalizar.

Estudiante 13: Podría poner un 2 acá en vez de un cero. (Señala el eje $y$ y el valor inicial que es cero).

Docente: No, toca buscar entre las opciones una que dice eso ... o otra que dice mostrar los datos, o sea que muestre los datos encima.

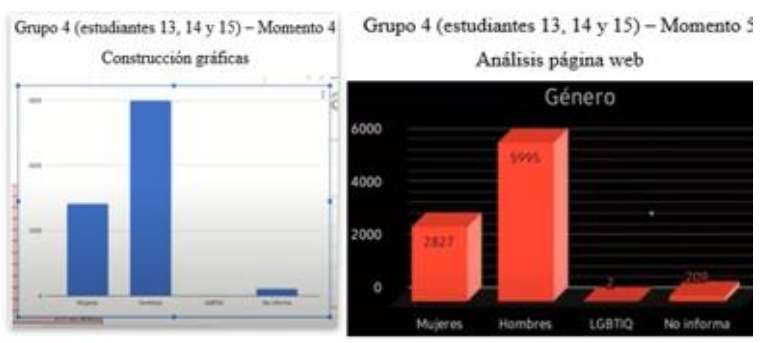

Grupo 1 (estudiantes 1, 2, 3 y 4)-Momento 5 - Análisis página web

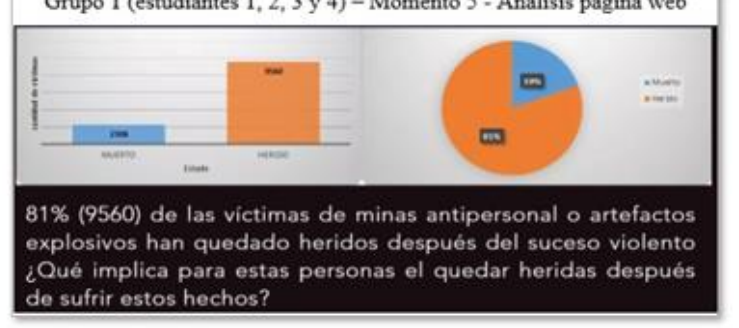

El estudiante 13 manifiesta "no me está marcando uno", lo que quiere decir que él se percata que la gráfica comunica información incorrecta, ya que según la gráfica dada parece no existir víctimas de personas LGBTI, aunque el programa realiza correctamente el gráfico pues la considerable diferencia entre las 5995 víctimas del género masculino frente a las 2 víctimas LGBTI, no permite que la barra relacionada con estas últimas tenga una altura notable, sin embargo, los estudiantes del grupo 4 lograron modificar las características visuales del gráfico de tal manera que se comunicara correctamente la información (Figura 5 - Grupo 4). Esta situación deja ver que la correcta comunicación de la información utilizando como herramienta objetos matemáticos, no requiere solamente de conocimientos escolares como "la construcción de gráficos estadístico según el tipo de variable", sino que implica una contextualización del problema que le dé sentido a lo representado, de tal forma que sea posible determinar si la información que se está presentando es la que se quiere comunicar. Como lo plantea el MEN (1998) el uso de tecnologías en la escuela enfatiza en la comprensión más que en la mecanización, en este caso la correcta construcción del gráfico de barras no es relevante frente a lo que los estudiantes quieren expresar a través de este. Además, expone cómo los recursos tecnológicos son una herramienta que permite modificar los recursos visuales, en este caso las características del gráfico de barras, de forma que se comunique correctamente la información.

En las presentaciones finales los estudiantes hicieron uso de diferentes objetos matemáticos para manifestar sus interpretaciones frente aspectos de cada modalidad de violencia, es el caso del grupo 1 quienes se apoyaron en cifras, porcentajes y gráficas para exponer el principal estado en el que quedan las víctimas de minas antipersonal, artefactos explosivos o municiones sin explotar (Figura 5 - Grupo 1).

En este caso, los estudiantes hacen uso de diferentes objetos matemáticos para comunicar que hay más víctimas que quedan heridas y centrar el interés en estas, aquí se identifica que inicialmente realizaron la lectura de la gráfica circular para resaltar el porcentaje de las personas heridas apoyándolo con la cantidad correspondiente, dato que obtienen del gráfico de barras; el uso de diferentes recursos matemáticos para resaltar información específica sobre el estado de las víctimas tiene como fin llamar la atención del espectador, para así abrir paso a su cuestionamiento sobre la vida que puede llevar una persona después de sufrir estos hechos. A partir de lo anterior se evidencia que el grupo 1 utiliza las matemáticas para hacer un llamado a la concientización sobre lo injusto que puede ser para una persona tener que afrontar la vida con lesiones físicas incurables. Como lo afirma Skovsmose y Valero (2012), en su tesis sobre la relación crítica 
entre matemáticas y democracia, depende del sentido que se les dé a las matemáticas el que sea posible consolidar la justicia social, en este caso, justicia para las personas que quedan heridas.

En relación con la competencia Razonar, resaltamos que en su mayoría los estudiantes realizaron sus presentaciones finales con argumentaciones basadas en lo aprendido y reflexionado durante el Escenario de Investigación.

\section{Figura 6}

Categoría razonar - Página web
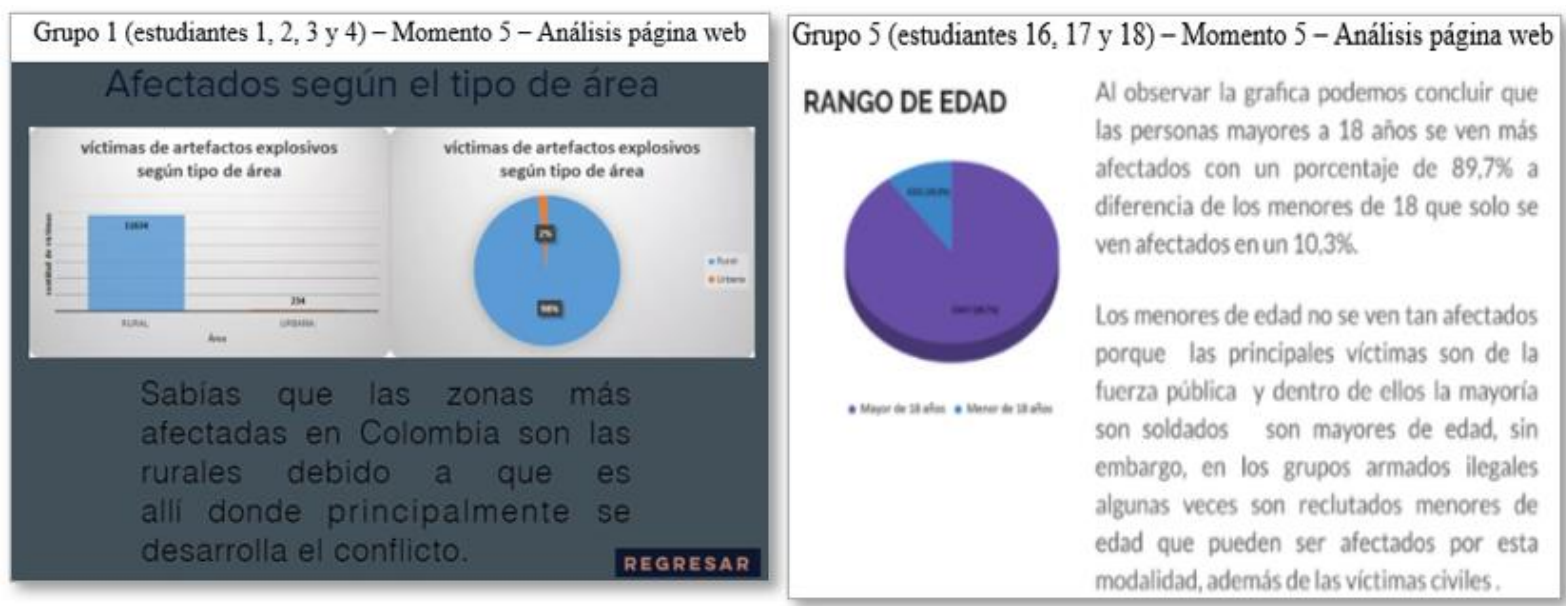

En las presentaciones de los grupos 1 y 5 se evidencia que los estudiantes realizaban la lectura de la gráfica e intentaban dar razones o justificaciones sobre porqué se obtienen estos resultados con base en los conocimientos que habían adquirido sobre el conflicto armado. El grupo 1 (Figura 6 - Grupo 1), que profundizó sobre la modalidad de minas antipersonal y artefactos explosivos, tiene claro que el conflicto armado se ha dado en todo el territorio colombiano, por ello, argumenta que las zonas rurales son las más afectadas por las minas antipersonal dado que también son las más vulneradas por todo el conflicto en general.

El grupo 5 (Figura 6 - Grupo 5), también profundizó sobre la modalidad de minas antipersonal, en la presentación relacionada con el rango de edad de las víctimas las estudiantes inicialmente exponen un párrafo introductorio en el que se realiza una lectura descriptiva de su gráfica para luego argumentar porqué se dan estos resultados; ellas manifiestan que "los principales afectados son la fuerza pública" es decir que reconocen que esta modalidad es usada usualmente por grupos armados ilegales con el fin de afectar a sus enemigos (GMH, 2013), entre ellos la fuerza pública, información que determinaron en otra de sus gráficas, para afirmar que las principales víctimas son "soldados" mayores de 18 años, esto teniendo en cuenta que solo los mayores de edad hacen parte de la fuerza pública; para el caso de los menores de edad, lo justifican como víctimas civiles o jóvenes que son reclutados por grupos armados ilegales y obligados a participar de la guerra, lo que hace reconocimiento a otras modalidades de violencia.

Las presentaciones de los grupos 1 y 5 develan que las conexiones entre la información obtenida durante el Escenario de Investigación les ofrece una cadena de argumentación que justifica porqué los resultados de las gráficas son estos y no otros, además, permite ver cómo los gráficos estadísticos les aportan información que conectada con sus conocimientos previos les amplía la comprensión sobre las modalidades de violencia, de tal forma que son capaces de opinar sobre las causas y consecuencias de las mismas; como afirma Gutiérrez (2013), utilizar las matemáticas como herramienta es una práctica humana con valor social que posibilita dar argumentos y juicios de verdad.

Respecto a la última competencia planteada por Alsina (2010), Representación, se evidenció que los estudiantes desde el primer momento lograban expresar sus pensamientos, opiniones o reflexiones centrados en recursos como la palabra, los mapas mentales, los cuadros organizativos y las imágenes.

En cuanto a las palabras se evidencia cómo desde el momento 1 se hace el uso de este recurso para expresar sus pensamientos y emociones; es el caso cuando en una discusión grupal las investigadoras preguntaron a los estudiantes qué hechos similares a los que vivieron los protagonistas de la película Pequeñas Voces conocían, los estudiantes 13, 19 y 21 a través de sus palabras relataron el acercamiento que habían tenido con el conflicto armado colombiano (Figura 7 - Transcripción de audio). 
Figura 7

Categoría representación - Transcripción de audio 24 de febrero y consulta estudiante

Momento 1 - Transcripción de audio 24 de febrero

Estudiante 21: (Habla con seguridad y confianza) Mi papá perteneció a las fuerzas armadas y hay un señor que hizo una pelicula sobre el conflicto armado, es verdad que hay mucha gente que pierde la vida, las extremidades, a él le dieron siete tiros y le perforaron los puimones, vive de milagro, no pudo hablar $n i$ caminar por mucho tiempo, se dio cuenta de muchas cosas y se logró recuperar, pero en medio de esto perdió muchas cosas.

Estudiante 13: (Habló tímidamente) Es una historia, pero cortita, bueno mi abuela cuando estaba pequeña ella vivía en el campo y llegaron los guerrilleros y le dijeron que se fuera, como no lo hicieron le mataron al papá y le dispararon a mi abuela, pero afortunadamente está viva.

Estudiante 19: (Tomó aire para empezar hablar) Eso fue cuando yo era una bebe y mi mamá trabajaba para solucionar conflictos entre el ELN y las FARC, las guerrillas entre comillas de esa época, y bueno pues, eso fue hace mucho tiempo, yo tenia un año de nacida y mi mamá contó que esa vez ella estaba ahi ayudando para que entre las dos guerrillas se pudieran arreglar razonando o haciendo varias cosas [...], hasta que un día alguien del mando de [...] no le hizo caso y empezó a estar en contra y secuestró a mi mamá, una enfermera y hasta una señora que estaba embarazada y bueno estaban muy angustiadas, $y$ mi mamá dijo tranquilas vamos a llamar a la policia, se demoraron como 20 minutos, en las noticias apareció eso, y salvaron a la enfermera, mi mamá estaba a punto de morir porque (rompió en llanto pero aun así lo terminó, durante el relato los compañeros más cercanos la miraron un poco consternados y los demás estaban desconcertados) uno de los policías la amenazó y dijo que sabía que su familia estaba conformada por mis abuelos y usted tiene una hija y la empezó amenazar y mi mamá dijo si va hacer algo hágamelo a mi pero a mi hija nunca me la toque.

Grupo 7 (estudiante 22) - Momento 3 - Búsqueda de información

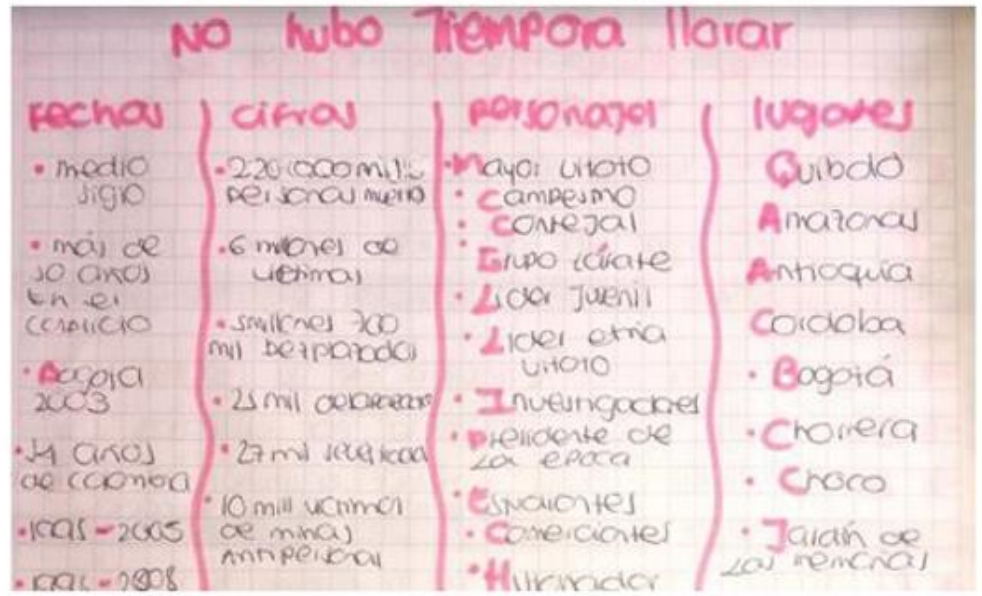

La intervención de estos tres estudiantes muestra la representación de la información por medio de la palabra, que no solo da a conocer los relatos, sino que transmite emociones y sentimientos a sus receptores, por ejemplo, el estudiante 13 al hablar tímidamente y manifestar "es una historia cortita" permite cuestionar si la razón de su comportamiento es por el tema que se está tratando o porque su historia es personal y no sabe cómo la asumirán sus compañeros, por ello intenta dar poco valor a su testimonio al cuantificarla a través de la palabra "cortita"; en cambio, la estudiante 21 transmite seguridad y confianza aseverando que sabe sobre el tema, en su afirmación "hay mucha gente que pierde la vida" quiere dar a entender a sus compañeros que esta problemática es real y aunque no conoce las cifras que sustenten su afirmación a través del adverbio de cantidad "mucho" espera llamar la atención de sus compañeros y concientizarlos que es un tema complejo.

Así mismo, la estudiante 19, aunque sabía que para ella no era fácil contar esta historia y le generaba sentimientos dolorosos, decide hacerlo, impactando a sus compañeros con sus palabras y expresiones, aunque ella afirma que "eso fue hace mucho tiempo" deja en el ambiente que el conflicto armado puede estar más cerca de lo que muchas veces se asume, que en verdad afecta a todos de alguna manera, además, que hay muchas cosas por aprender y reflexionar. Lo vivido en esta sesión también es evidencia de que el Escenario de investigación se desarrollaba en un ambiente confiable y fortalecido por el aprendizaje dialógico ya que "se escucha a los aprendices; mostrando (y sintiendo) interés por sus puntos de vista, por sus concepciones, y por sus intuiciones" (Guerrero, 2008, p. 6).

En cuanto a los mapas mentales y cuadros organizativos, estos fueron utilizados por los estudiantes a medida que requerían organizar la información que iban obteniendo en el transcurso del Escenario de Investigación para clarificar y conectar sus aprendizajes, por ejemplo, los apuntes realizados por la estudiante 22 mientras veían el documental "No hubo tiempo para la tristeza" (Figura 7 - Consulta estudiante).

En los registros de la estudiante se hace claridad en distintos tipos de información como dónde, cuándo, cómo o por qué ocurren estos hechos, que al relacionarla con las cifras que obtenían en las bases de datos, logra profundizar en sus presentaciones finales sobre las consecuencias de las modalidades de violencia para 
las víctimas. Lo cual evidencia que la representación de la información por medio de cuadros o mapas mentales que tengan una estructura lógica que entrelace o conecte los datos permite que se determine información importante: principales causas del conflicto, periodos de violencia, cifras de víctimas, lugares donde se ha desarrollado o actores participantes, de tal modo que amplían su comprensión ante las modalidades de violencia.

Finalmente, se destaca el uso de la imagen como representación que a pesar de no contener cifras o texto transmite información que impacta y conecta con la temática tratada, por ejemplo, en una de las conversaciones del grupo 1 mientras organizaban su página web se manifestó lo importante de la imagen para lo que querían transmitir (Figura 8 - Grupo 1).

En este diálogo, el estudiante 4 opina que la imagen a seleccionar debe ser "trágica porque es la cantidad de muertos", es decir que la imagen debe representar algún hecho desastroso que genere impacto y comunique por sí misma que la cantidad de muertos es alta. Así como el grupo 1, otros grupos también se apoyaron de las imágenes para destacar la información que querían presentar (Figura 8 - Grupo 4 y 5).

\section{Figura 8}

Categoria representación - Transcripción de audio 3 de agosto y página web

Grupo 1 -- Momento 4 - transcripción audio 3 de agosto

Estudiante 1: Porque no le cambias esa imagen.

Estudiante 4: ¿Por cuál?

Estudiante 1: No sé.

Estudiante 4: No sé, toca una trágica porque es la cantidad de muertos.

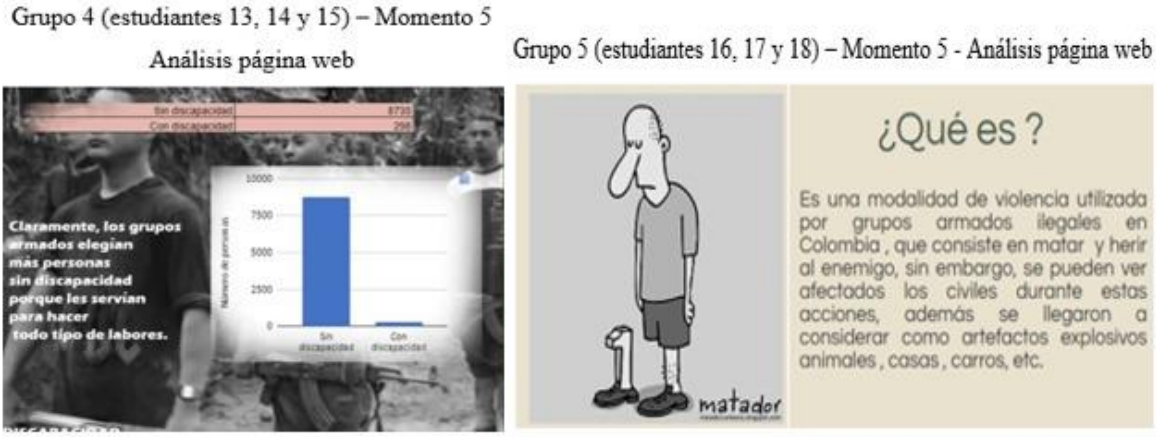

En la figura 8 se presenta el trabajo del grupo 4, en este los estudiantes apoyan su presentación con un fondo real que contextualiza al espectador sobre lo que deben vivir las víctimas del reclutamiento ilícito; además, se evidencia como el grupo 5 no utilizó una imagen real, sino que acompañó la definición de la modalidad de violencia sobre minas antipersonal con una caricatura que expresa tristeza y da al espectador el sentir de tener una extremidad ausente, efectivamente es una imagen que no es escandalosa ni exagera la realidad de las víctimas, sino que da una invitación para ponerse en el lugar de ellas. Lo anterior es evidencia de cómo los estudiantes reconocen que una imagen representa y transmite información por sí sola, pero que, acompañada con algunas cifras o palabras, despierta algunas emociones y permite generar cuestionamientos o reflexiones a quien la observa.

Teniendo en cuenta las evidencias presentadas en cada una de las categorías de análisis relacionadas con el desarrollo de competencias matemáticas asociadas con competencias ciudadanas cognitivas, comunicativas y emocionales, se da cuenta de las matemáticas como herramienta que aplicada a contextos sociales reales permite que los estudiantes reflexionen, comprendan, amplíen y comuniquen su conocimiento frente al conflicto armado colombiano, dándoles la oportunidad de cuestionar por qué se vive esta violencia en el país o qué pasa con la vida de las víctimas, además de ofrecer la posibilidad de dar opiniones informadas por medio de distintos recursos expresivos en los cuales se develen las injusticias sociales, en este caso las injusticias con las víctimas de cada modalidad.

Considerando que a través de esta investigación se buscaba el desarrollo de competencias ciudadanas y matemáticas para la comprensión de algunas modalidades de violencia del conflicto armado colombiano, a partir de las evidencias presentadas y acorde con lo propuesto por el documento de Formación Ciudadana (Ruiz y Chaux, 2005), los Estándares Básicos de Competencias Ciudadanas (MEN, 2006), las Competencias Matemáticas del Ciudadano de Alsina (2010) y la perspectiva de la Educación Matemática Crítica (Skovsmose, 2000), se demuestra cómo a través de la planeación y ejecución de un Escenario de Investigación Real se potencian estas competencias en pro de la comprensión de las modalidades de la 
violencia, de forma que los estudiantes toman conciencia sobre sus impactos, manifiestan preocupación por las víctimas y piensan en alternativas para la no repetición.

Muestra de lo anterior son las reflexiones finales de los estudiantes, en las que emergen características propias de un ciudadano crítico, como la estudiante 8 , quien expresa "de ahora en adelante tendré presentes a todas esas víctimas" y "cuando tenga la edad requerida para votar intentaré escoger representantes que ayuden a lograr la paz en Colombia", constituyéndola como un actor político que toma decisiones y proyecta intervenir en asuntos de interés público; o el estudiante 10, quien afirma que "en nuestras manos está cambiar nuestro país" y "hoy pude aportar ese granito de arena que todos deberíamos de aportar", destacando su sentido de pertenencia con Colombia y adquiriendo deberes con la sociedad como "mostrar a muchas más personas la realidad de nuestro país para que así todos podamos ayudar".

\section{Discusión y conclusiones}

Teniendo en cuenta que la guerra en Colombia representa una problemática político-social que por años ha afectado a miles de colombianos $(\mathrm{GMH}, 2013)$ y que la educación matemática debe propender por el desarrollo de competencias matemáticas para afrontar la realidad social (MEN, 1998), la principal conclusión del estudio que derivó este artículo es precisamente que las matemáticas son una herramienta poderosa para develar injusticias y desigualdades presentes en este conflicto (Millán y Vernaez, 2005). De manera que se demuestra que puede construirse una relación entre la comprensión del conflicto armado en la escuela y el conocimiento matemático, pues a través del análisis, cuestionamiento y reflexión sobre la información de dominio público se hace evidente los impactos, magnitud y degradación de la guerra, contribuyendo con ello a la formación ciudadana crítica.

Es así como desde la perspectiva de la Educación Matemática Crítica se aportó en el diseño, gestión y evaluación de un Escenario de Investigación que centró su interés en la comprensión de algunas modalidades de violencia del conflicto armado colombiano y su impacto en las víctimas. De modo que en la aplicación del Escenario se promovió el desarrollo de competencias matemáticas en los estudiantes, que permitieron emerger características propias de un ciudadano crítico, el cual acepta que tiene derechos y deberes que debe ejercer para el bien de todos los ciudadanos, se compromete a actuar reconociendo sus derechos en la toma de decisiones, participa en asuntos de interés público, y asume sus deberes con la sociedad colombiana, de forma que reclama justicia ante la desigualdad social, política, económica o cultural, reflexiona críticamente sobre las consecuencias de la guerra y es empático con las víctimas.

A través de esta experiencia, se reconoce el papel que representan los docentes de matemáticas en cuanto a la formación ciudadana, pues ellos pueden brindar la posibilidad de abordar problemáticas sociales, políticas, culturales y económicas en el aula, además de no renunciar a dotar a los estudiantes de conocimientos matemáticos necesarios que les permita entender la complejidad del mundo que les rodea. Por tanto, es importante que los profesores asuman las matemáticas como una herramienta poderosa que posibilita a las personas participar democráticamente, emprender acciones de transformación y argumentar, reflexionar, razonar, comunicar o representar aspectos de situaciones problema. Sin embargo, para ello se debe promover un uso adecuado de estos conocimientos y habilidades, lo que implica promover el desarrollo de competencias ciudadanas que garanticen la formación de un ciudadano crítico que usa las matemáticas para el bien común.

A partir de lo anterior, se considera pertinente, para futuras investigaciones, pensar en cuáles son los requerimientos que exige la formación de docentes en matemáticas para que se perfilen como docentes interdisciplinares, pues esta propuesta logró trabajar aspectos del conflicto armado colombiano estableciendo un vínculo entre la formación ciudadana, la enseñanza de las ciencias sociales y los conocimientos matemáticos, sin embargo, existen múltiples espacios en la escuela o fuera de ella donde las matemáticas pueden contribuir a la comprensión de diferentes asuntos, es así como, surgen las siguientes preguntas: ¿por qué estas propuestas no son tan comunes?, ¿los docentes de matemáticas deben ser polímatas para intervenir en otras áreas? y ¿son las dinámicas de la escuela las que deben cambiar?

\section{Referencias}

Alsina, C. (2010). Matemáticas para la ciudadanía. En M. Callejo y J. Goñi (Ed), Educación matemática y ciudadania (pp. 89-102). Grao. 
Ayala, B (2019). Formación ciudadana crítica: una aproximación desde el desarrollo de competencias estadísticas y la resolución de problemas -rp-. Tesis de Maestría inédita. Repositorio institucional de la Universidad Distrital Francisco José de Caldas. http://hdl.handle.net/11349/15331

Álvarez, I. y Montoya, D. (2011). Ambientes de aprendizaje y cultura estadística a través de un experimento de enseñanza para estudiantes de grado noveno. Tesis de Maestría inédita. Repositorio institucional de la Universidad Pedagógica Nacional. http://hdl.handle.net/20.500.12209/234

Amaya, L. (2018). Procesos de inclusión en contextos de re-educación: alternativas desde la educación matemática. Tesis de Maestría inédita. Repositorio institucional de la Universidad Distrital Francisco José de Caldas. http://hdl.handle.net/11349/14303

Angulo, E. y Solano, J. (2013). Educación Matemática Crítica y Ambientes de Aprendizaje. Posibilidades y dificultades en un proyecto de formación de estudiantes críticas. Tesis de Maestría inédita. Repositorio institucional de la Universidad Pedagógica Nacional http://hdl.handle.net/20.500.12209/228

Arias, D. (2015). La enseñanza de las ciencias sociales en Colombia. Lugar de las disciplinas y disputa por la hegemonía de un saber. Revista Estudios Sociales, 52, 134-146.

Chaparro, M. (2019). La creación de escenarios educativos de aprendizaje de las matemáticas, como una posibilidad de inclusión y participación igualitaria. El caso de la inequidad entre hombres y mujeres. Tesis de Maestría inédita. Repositorio institucional de la Universidad Distrital Francisco José de Caldas. http://hdl.handle.net/11349/15582

D’Ambrosio, U. (2007). La matemática como ciencia de la sociedad. En J. Giménez, F. Díez y M. Civil (Ed), Educación matemática y exclusión (pp. 83-102). Grao.

Guerrero, C. (2008). Educación matemática crítica. Influencias teóricas y aportes. Evaluación e Investigación, $1(3), 1-14$.

Gutiérrez, R. (2013). The Sociopolitical Turn in Mathematics Education. Journal for Research in Mathematics Education, 44(1), 37-68. DOI: http://doi.org/10.5951/jresematheduc.44.1.0037

Grupo de Memoria Histórica-GMH. (2013). ¡BASTA Y A! Colombia: Memorias de guerra y dignidad. Imprenta Nacional.

Goñi, J. (2010) La aspiración a la ciudadanía y el desarrollo de la competencia matemática. En M. Callejo y J. Goñi (Ed), Educación matemática y ciudadania (pp. 11-58). Grao.

González, J. y Tovar, J. (2017). Propuesta para promover la educación estadística crítica en estudiantes de secundaria a través de la cultura mediática. Tesis de Maestría inédita. Repositorio institucional de la Universidad Pedagógica Nacional. http:/ / hdl.handle.net/20.500.12209/9454

Gómez, E. (2016). Ciudadanía Ambiental desde las prácticas de medición en la construcción del espacio de reforestación. Tesis de Maestría inédita. Repositorio institucional de la Universidad Pedagógica Nacional. https://doi.org/10.17227/bio-grafia.extra2016-6320

Hernández, R. (2014). Metodología de la investigación. McGRAW-HILL.

Lizarralde, M. (2012). Escuela y guerra, la invisibilidad de los ambientes educativos. En B. García (Ed.). Violencia y educación. Historia de la Educación, la Pedagogía y la Educación Comparada (pp. 33-70). Doctorado Interinstitucional en Educación Universidad Distrital Francisco José de Caldas.

Millán, L. y Vernaez, G. (2005). Sobre educación matemática y su relación con lo político y social. En D. Mora, Didáctica crítica, educación crítica de las matemáticas y etnomatemática: perspectivas para la transformación de la educación matemática en América Latina (pp. 315-336). Campo Iris.

Ministerio de Educación Nacional-MEN. (1998). Lineamientos Curriculares en Matemáticas. Bogotá, Colombia.

Ministerio de Educación Nacional-MEN. (2006). Estándares Básicos de Competencias Ciudadanas. Formar para la ciudadanía si es posible. Bogotá, Colombia.

Pagés, J. (2009). Enseñar y aprender ciencias sociales en el siglo XXI: reflexiones casi al final de una década. En Investigación en Educación, Pedagogía y Formación Docente, II Congreso Internacional (p. 140-154).

Romero, M. (2012). Conflicto armado y Escuela en Colombia. En B. García (Ed.), Violencia y educación. Historia de la Educación, la Pedagogía y la Educación Comparada (pp. 13-32). Doctorado Interinstitucional en Educación Universidad Distrital Francisco José de Caldas.

Ruiz, A. y Chaux, E. (2005). Formación ciudadana. Ascofade.

Scaglia, S. (2012). Educación Matemática Crítica. En M. Pochulo y M. Rodríguez (Eds.), Educación Matemática aportes a la formación docente desde distintos enfoques teóricos (pp. 201-226). Editorial Universitaria Villa María.

Skovsmose, O. (1999). Hacia una filosofía de la educación matemática crítica. Ediciones Uniandes.

Skovsmose, O. (2000). Escenarios de Investigación. Revista EMA, 6(1), 3-26.

Skovsmose, O. y Valero, P. (2012). Rompimiento de la neutralidad política: El compromiso crítico de la educación matemática con la democracia. En O. Skovsmose y P. Valero (Ed.), Educación Matemática Crítica. Una visión sociopolítica del aprendizaje y la enseñanza de las matemáticas (pp. 1-23). Ediciones Uniandes. 
Torres, E. (2015). Narratividad y tiempo: niños y niñas desvinculados del conflicto armado colombiano. En D. Calderón (Ed.), Lenguaje, cultura e investigación: problemas emergentes en educación (pp. 59-82). Doctorado Interinstitucional en Educación Universidad Distrital Francisco José de Caldas.

Torres, C. (2016). Posibilidades y dificultades de los ambientes de aprendizaje construidos a partir de los fenómenos de conducción eléctrica. Un estudio en la Educación Media Especializada. Tesis de Maestría inédita. Repositorio institucional de la Universidad Pedagógica Nacional. http://hdl.handle.net/20.500.12209/228

Valero, P. (2006). ¿De carne y hueso? La vida social y politica de las competencias matemáticas [Memorias]. Foro Educativo Nacional de Colombia. https://redaprende.colombiaaprende.edu.co/metadatos/recurso/de-carne-y-hueso-la-vida-social-ypolitica-de-la-c/

Zamora, M. (2018). Razonamiento crítico en representaciones gráficas estadísticas desde el ambiente económico de la unidad familiar del estudiante. Tesis de Maestría inédita. Repositorio institucional de la Universidad Del Valle. http://hdl.handle.net/10893/12347 